\title{
Low-energy charged particle measurement by MAP-PACE onboard SELENE
}

\author{
Yoshifumi Saito $^{1}$, Shoichiro Yokota $^{1}$, Kazushi Asamura $^{1}$, Takaaki Tanaka ${ }^{1}$, Ryota Akiba ${ }^{2}$, Masaki Fujimoto $^{1}$, \\ Hiroshi Hasegawa ${ }^{1}$, Hajime Hayakawa ${ }^{1}$, Masafumi Hirahara ${ }^{3}$, Masahiro Hoshino ${ }^{3}$, Shinobu Machida ${ }^{4}$, \\ Toshifumi Mukai ${ }^{1}$, Tsugunobu Nagai ${ }^{5}$, Tsutomu Nagatsuma ${ }^{6}$, Masato Nakamura ${ }^{1}$, \\ Koh-ichiro Oyama ${ }^{1}$, Eiichi Sagawa ${ }^{7}$, Susumu Sasaki ${ }^{1}$, \\ Kanako Seki ${ }^{8}$, and Toshio Terasawa ${ }^{5}$

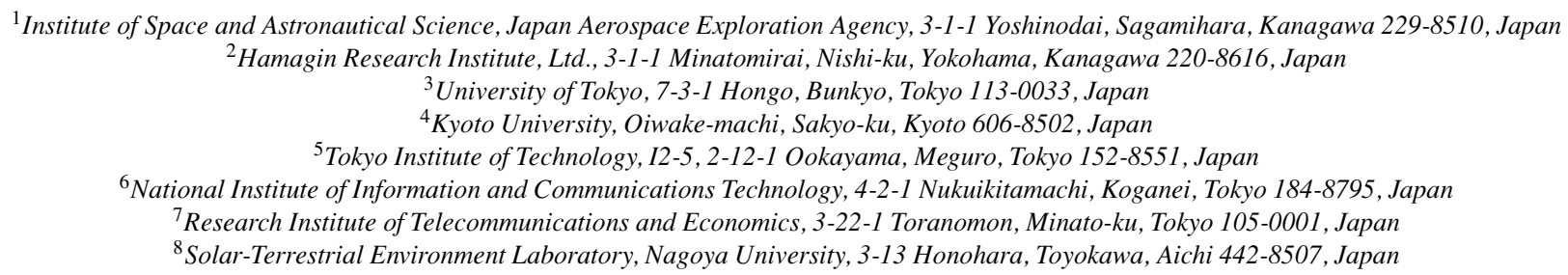

(Received March 20, 2007; Revised October 8, 2007; Accepted November 8, 2007; Online published April 9, 2008)

MAP-PACE (MAgnetic field and Plasma experiment-Plasma energy Angle and Composition Experiment) is one of the scientific instruments onboard the SELENE (SELenological and ENgineering Explorer) satellite. PACE consists of four sensors: ESA (Electron Spectrum Analyzer)-S1, ESA-S2, IMA (Ion Mass Analyzer), and IEA (Ion Energy Analyzer). ESA-S1 and S2 measure the distribution function of low-energy electrons below $15 \mathrm{keV}$, while IMA and IEA measure the distribution function of low energy ions below $28 \mathrm{keV} / \mathrm{q}$. Each sensor has a hemispherical field of view. Since SELENE is a three-axis stabilized spacecraft, a pair of electron sensors (ESA-S1 and S2) and a pair of ion sensors (IMA and IEA) are necessary for obtaining a three-dimensional distribution function of electrons and ions. The scientific objectives of PACE are (1) to measure the ions sputtered from the lunar surface and the lunar atmosphere, (2) to measure the magnetic anomaly on the lunar surface using two ESAs and a magnetometer onboard SELENE simultaneously as an electron reflectometer, (3) to resolve the Moon-solar wind interaction, (4) to resolve the Moon-Earth's magnetosphere interaction, and (5) to observe the Earth's magnetotail.

Key words: Moon, plasma, magnetic anomaly, alkali atmosphere, magnetosphere, solar wind.

\section{Introduction}

Low-energy charged particles around the Moon were vigorously observed by Moon orbiting satellites and plasma instrumentation placed on the lunar surface in 1960s and 1970s (Lyon et al., 1967; Colburn et al., 1967; Anderson et al., 1972; Howe et al., 1974; Neugebauer et al., 1972; Clay et al., 1972; Hills et al., 1972). Many new discoveries concerning the lunar plasma environment were made during this period. Though there were some satellites that explored the Moon afterwards, most were dedicated to the global mapping of the lunar surface (Nozette et al., 1994; Binder, 1998; Foing et al., 2006). Except the low-energy electron measurement by Lunar Prospector (Binder, 1998), the lunar wake plasma data obtained by WIND during its Moon fly-by (Ogilvie et al., 1996), and reports on remote detection of the lunar ions (Hilchenbach et al., 1993; Mall et al., 1998; Futaana et al., 2003) and lunar electrons (Futaana et al., 2001) there has been almost no new information about the low-energy charged particles around the Moon.

SELENE is a Japanese lunar orbiter that will study the

Copyright (c) The Society of Geomagnetism and Earth, Planetary and Space Sciences (SGEPSS); The Seismological Society of Japan; The Volcanological Society of Japan; The Geodetic Society of Japan; The Japanese Society for Planetary Sciences; TERRAPUB. origin and evolution of the Moon by means of global mapping of element abundances and mineralogical composition, and surface geographical mapping from an altitude of $100 \mathrm{~km}$. SELENE was successfully launched on 14 September 2007 by the HIIA launch vehicle from Tanegashima Space Center in Japan. It was inserted into a circular lunar polar orbit of $100 \mathrm{~km}$ altitude and will continue observation for at least 1 year. Fourteen scientific instruments are onboard the three-axis stabilized spacecraft SELENE; one of the panels is always facing the lunar surface. One of the scientific instruments, PACE, was developed for the comprehensive three-dimensional plasma measurement around the Moon. After describing the science objectives of PACE in Section 2, we go on to present the instrumentation, instrument calibration, observation mode of PACE, and ground science data processing in Sections 3, 4, 5, and 6. In Section 7, we discuss the expected observation of lunar alkali atmosphere and lunar surface magnetic anomaly.

\section{Science Objectives of PACE}

2.1 Ions originated from the lunar surface and the lunar atmosphere

Ground-based observations carried out at the end of the 1980s revealed the existence of tenuous alkali-atmosphere 
around the Moon. Potter and Morgan (1988) discovered the existence of $\mathrm{Na}$ and $\mathrm{K}$ atmospheres above the sunlit limb of the Moon for the first time. Since then, several generation mechanisms have been proposed for the rarefied lunar alkali-atmosphere (Stern, 1999). Sputtering caused by the solar wind ions has drawn considerable attention, because it produces secondary particles that reflect the lunar surface composition. On the other hand, Potter et al. (2000) found that solar photons play a dominant role in desorption of the lunar alkali atmosphere. According to these researchers, sputtering of the lunar surface only contributes to the creation of the lunar $\mathrm{Na}$ atmosphere by enhancing the diffusion of $\mathrm{Na}$ to the lunar surface. Sputtered or desorped particles from the lunar surface are mainly composed of neutrals, which are ionized by solar photons and electrons. Both ionized particles and sputtered/desorped ions are accelerated and transported by the solar wind in cycloidal motion. These ions will be observed by the in-situ low-energy ion measurement by PACE. PACE will also remotely reveal the global composition of the lunar surface by detecting ions sputtered by the solar wind and tracing them back to their sputtering point in the same way as laboratory Secondary Ion Mass Spectrometry (SIMS) (Elphic et al., 1991). The research of the lunar atmosphere and lunar surface material is one of the most important aims of PACE.

\subsection{Magnetic anomalies on the lunar surface}

The solar wind electrons and the magnetotail electrons that reach the Moon will be absorbed if there is no magnetic field on the lunar surface. However, with the existence of the remnant magnetic field on the Moon, the electrons moving with a large angle around the ambient magnetic field will be mirror-reflected back to SELENE. Measuring the pitch angle distribution of the reflected electrons, the remnant magnetic field on the lunar surface can be deduced (Howe et al., 1974; Anderson et al., 1975). The previous remnant magnetic field measurement using mirror reflected electrons were conducted by the Apollo 15, 16 sub-satellites whose orbits were limited around the equator region of the Moon (Anderson et al., 1977; Anderson and Wilhelms, 1979). Lunar Prospector also measured remnant magnetic field on various areas of the lunar surface using electron reflectometer (Lin et al., 1998; Halekas et al., 2001). The PACE-ESA sensors, due to the $90^{\circ}$ orbit inclination of the SELENE main orbiter, will survey the remnant magnetic field on almost all the lunar surface with higher spatial resolution than previous electron reflectometer measurements. The detection of magnetic anomalies on the lunar surface is another important aim of PACE.

\subsection{Moon-solar wind interaction}

As widely accepted, there is almost no intrinsic magnetic field around the Moon. Thereby, when the Moon is in the solar wind, there is no well-defined bow shock as can be found in the terrestrial magnetosphere. Alternatively, the void region of plasma surrounded by the rarefaction region is created just behind the Moon. The lunar wake consists of these void and rarefaction regions. In the rarefaction region, the plasma is in a highly turbulent state, and various kinds of waves are observed and particles are accelerated and heated. We regard the lunar wake as a suitable and ideal region to study the formation of plasma turbulence. It is also known that there is some plasma compression at the limb of the Moon and the degree of the plasma compression has a regional dependence (Criswell, 1973). It is therefore thought that there is a weak but significant magnetic field anomaly on the surface of the Moon and that this deflects the solar wind particles and varies the degree of compression (Lin et al., 1998). It is of primary importance to study the structure of the lunar wake and the behavior of plasma near the limb of the Moon. The measurements of PACE will reveal more details of such phenomena and further verify proposed models of the solar wind and Moon interaction by conducting three-dimensional plasma particle measurement, obtaining their velocity momentum, i.e., the number density, the velocity, and the temperature.

\subsection{Moon-Earth's magnetosphere interaction}

In the Earth's plasma sheet, at a downtail distance of $\sim 60 R_{E}$, which corresponds to the Moon orbital distance, Earthward flowing plasmas, tailward flowing plasmas, and counter-streaming (Earthward and tailward) flows often exist. They are a mixture of solar wind origin and of ionospheric origin. It is very important to know the origins and dynamics of these plasmas. With this knowledge, we can understand the formation mechanism of the magnetosphere and the structure of the magnetotail. The Moon provides an excellent experimental tool for such research. In the first approximation, the Moon is not any obstacle for the magnetic fields. However, the Moon is a strong absorber of plasmas. The Moon provides "an eclipse" of the Earth's magnetotail. When the Moon is in the magnetotail, plasmas from the distant tail cannot reach any observer on the Earthward side of the Moon, whereas plasmas from the Earth cannot reach any observer on the tailward side of the Moon. Therefore, PACE will be able to discriminate the tailward-flowing plasmas from the Earthward-flowing plasmas. It will be possible to correctly identify the tailward-flowing component and the Earthward flowing component in the counterstreaming flows. Furthermore, it will be possible to identify some 'hidden' plasmas in the flowing plasmas.

\subsection{Plasma measurement of the Earth's magnetotail}

A comprehensive plasma measurement near the Moon's orbit has been carried out only with the spacecraft Geotail. Geotail mainly provided a survey of plasmas in the SunEarth direction, not in the cross section of the magnetotail. A spacecraft on orbit around the Moon makes a detailed survey of plasmas in the cross section of the magnetotail, so that we would be able to examine various boundaries in the magnetotail (i.e., the distant bow shock, the magnetopause, the magnetopause boundary layer, the low-latitude boundary layer, the plasma sheet-tail lobe boundary). In the magnetotail, various plasma populations are controlled by the interplanetary magnetic field (IMF). The loading of ionospheric $\mathrm{O}^{+}$in the magnetotail depends strongly on the IMF. By measuring $\mathrm{O}^{+}$distribution in the tail cross section, we will be able to understand the loading and transport mechanisms of ionospheric ions in the tail lobe/mantle. The draping of the magnetic field lines near the magnetopause and the deformation of the magnetotail will be correctly evaluated in order to understand changes in the magnetotail structure. Furthermore, it will be possible to find the magnetic reconnection process in the tail magnetopause region. 


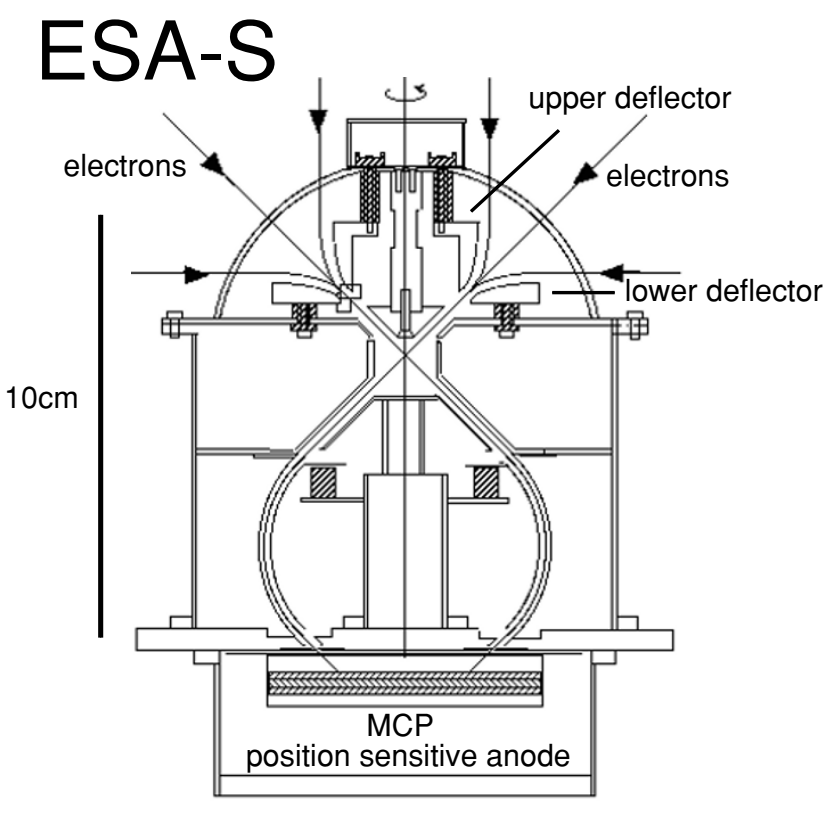

Fig. 1. Cross section of ESA-S1 and ESA-S2.

\section{Instrumentation}

\subsection{ESA-S1 and ESA-S2}

The ESA (Electron Spectrum Analyzer) sensor basically utilizes a method of a top hat electrostatic analyzer with angular scanning deflectors at the entrance and toroidal electrodes inside (see Fig. 1). The FOV (Field Of View) is electrically scanned between $\pm 45^{\circ}$ around the center of the FOV, that is $45^{\circ}$ inclined from the axis of symmetry. With two ESA sensors that are installed on the $+\mathrm{Z}$ (ESA-S1: looking down the lunar surface) and $-\mathrm{Z}$ (ESA-S2: looking against the lunar surface) panels of the spacecraft, the threedimensional electron distribution function is observed. The curved upper and lower angular deflectors are supplied with high voltage, swept between $0 \mathrm{~V}$ and $+4 \mathrm{kV}$. The inner toroidal electrode is also supplied with high voltage swept between $0 \mathrm{~V}$ and $+3 \mathrm{kV}$ simultaneously with the angular scanning deflectors. The electrons coming through the angular scanning deflectors are attracted down toward the inner electrode by the action of the applied potential. Only the electrons with a specific energy range can further travel down to the exit of the electrodes. We also placed a slit and a grid, supplied with slightly negative voltage $(-2.5$ to $-10 \mathrm{~V})$, to reflect the secondary electrons that give spurious counts. The electrons passing through the electrode enter the Micro-Channel Plate (MCP) and are intensified to detectable charge pulses. Finally, the charge pulses are received by one-dimensional circular resistive anode. The positions where the charge pulses are detected correspond to the incident azimuthal directions of the electrons. Tables 1 and 2 summarize the specifications of ESA-S1 and ESA-S2, respectively. Since the gap between the toroidal deflectors of ESA-S1 is larger than that of ESA-S2, the maximum energy and energy resolution of ESA-S1 is lower than ESA-S2 while the g-factor of ESA-S1 is larger than ESA-S2.
Table 1. Specifications of ESA-S1.

\begin{tabular}{lc}
\hline Energy range & $5 \mathrm{eV}-10 \mathrm{keV}$ \\
\hline Energy resolution & $15 \%(\mathrm{FWHM})$ \\
\hline Energy sweep step & 32 \\
\hline Field of view & $2 \pi \mathrm{str}$. \\
\hline FOV sweep range & $45^{\circ} \pm 45^{\circ}(\mathrm{Pol})$ \\
\hline Angular resolution & $5^{\circ}(\mathrm{Pol}) \times 8^{\circ}(\mathrm{Az})(\mathrm{FWHM})$ \\
\hline g-factor $\left(5^{\circ} \times 22.5^{\circ}\right)$ & $\mathrm{cm}^{2} \mathrm{str} \mathrm{keV} / \mathrm{keV}$ \\
$($ efficiency is not included $)$ & \\
\hline Time resolution & Toroidal analyzer \\
\hline Analyzer type & $30 \mathrm{~mm}$ \\
Average radius & $5 \mathrm{~mm}$ \\
Gap & $5 \mathrm{~mm}$ \\
Eccentricity &
\end{tabular}

Table 2. Specifications of ESA-S2.

\begin{tabular}{lc}
\hline Energy range & $5 \mathrm{eV}-15 \mathrm{keV}$ \\
\hline Energy resolution & $10 \%(\mathrm{FWHM})$ \\
\hline Energy sweep Step & 32 \\
\hline Field of view & $2 \pi$ str. \\
\hline FOV sweep range & $45^{\circ} \pm 45^{\circ}(\mathrm{Pol})$ \\
\hline Angular resolution & $5^{\circ}(\mathrm{Pol}) \times 8^{\circ}(\mathrm{Az})(\mathrm{FWHM})$ \\
\hline g-factor $\left(5^{\circ} \times 22.5^{\circ}\right)$ & $2 \times 10^{-4} \mathrm{~cm}^{2} \mathrm{str} \mathrm{keV} / \mathrm{keV}$ \\
$($ efficiency is not included $)$ & \\
\hline Time resolution & $1 \mathrm{~s}$ \\
\hline Analyzer type & Toroidal analyzer \\
Average radius & $30 \mathrm{~mm}$ \\
Gap & $3 \mathrm{~mm}$ \\
Eccentricity & $5 \mathrm{~mm}$ \\
\hline
\end{tabular}

\subsection{IMA and IEA}

The IMA (Ion Mass Analyzer) sensor consists of an energy analyzer that has the similar structure as the ESA sensors and an LEF (Linear Electric Field) TOF (Time Of Flight) ion mass analyzer (McComas and Nordholt, 1990; Yokota et al., 2005; Yokota and Saito, 2005) (see Fig. 2). The IEA (Ion Energy Analyzer) sensor consists of only an energy analyzer that is the same as the energy analyzer of IMA (see Fig. 3). IMA is installed on the $+Z$ (looking down the lunar surface) panel while IEA is installed on the $-Z$ (looking against the lunar surface) panel. The curved upper and lower angular deflectors of the energy analyzer are supplied with high voltage, swept between $0 \mathrm{~V}$ and $+5 \mathrm{kV}$. The inner spherical electrode is also supplied with high voltage swept between $0 \mathrm{~V}$ and $-4 \mathrm{kV}$ simultaneously with the angular scanning deflectors. A pair of electrodes are located between the spherical electrode and the angular deflectors; these serve as sensitivity control electrodes. Since the flux of the solar wind ions and that of the lunar-origin ions differs significantly, the sensitivity of the energy analyzer should be reduced continuously to about $1 / 100$ for the solar wind ion observation. The ions transmitted through the energy analyzer of IEA are detected by MCP with a one-dimensional circular resistive anode. The ions transmitted through the energy analyzer of IMA are post accelerated by $-15 \mathrm{kV}$ and enter into the LEF TOF mass analyzer part. Thin carbon foil $\left(0.5 \mu \mathrm{g} / \mathrm{cm}^{2}\right)$ is placed 
at the entrance of the LEF TOF mass analyzer, which generates start electrons when the ions pass through the carbon foil. The start electrons are accelerated by the electric field inside the mass analyzer and their positions are detected by one-dimensional circular resistive anode that is placed behind the MCP. These start electrons also generate start signals when they pass through a grid anode that is placed between the position-sensitive anode and the MCP. Most of the ions that pass though the carbon foil lose their initial charge state and enter into the mass analyzer as neutral particles. These neutral particles are detected by an anode that is in the center of the position-sensitive anode. These signals are used as stop signals. The mass/charge of the incident ions can be calculated from its energy/charge and the time of flight. The relation between the time of flight $t$ and the mass/charge $m / q$ for neutral particles is expressed as $m / q=2 / L^{2}\left(E / q+V_{\text {acc }}\right) t^{2}$, where $L$ is the flight path length of the neutral particles, and $V_{\text {acc }}$ is the post-acceleration voltage. Some of the incident ions enter the time-of-flight section as ions. These ions are reflected by the linear electric field whose intensity is proportional to the distance from the entrance point. The reflected ions generate secondary electrons when they collide with the top part of the mass analyzer. These electrons are accelerated and detected by the center anode, a process which generates stop signals. The relation between the time of flight $t$ and the mass/charge $m / q$ for ions is expressed as $m / q=t^{2} / \pi^{2} a$, where $a$ is the linear electric field gradient. Since time of flight of the reflected ions is proportional to the square root of the ion mass, the mass of the incident ions can be determined precisely without being affected by the angular scattering and the energy degradation caused by the ion passage in the carbon foil (McComas and Nordholt, 1990; Yokota et al., 2005). For the ions, the time-of-flight section of IMA acts as the so-called "isochronous time-offlight". With this design of LEF-TOF mass spectrometer, the center anode detects both neutrals (direct time-of-flight) and secondary electrons from the top part of the mass analyzer, generated by ions (isochronous time-of-flight). We have measured the time profile of several ion species by injecting those ions into the IMA. Each ion species has its own characteristic TOF profile, including neutral peak, ion peak, and/or negative ion peak (Yokota et al., 2005). Since some of these TOF profile overlap with each other, we will have to use a deconvolution tool made by using calibration data of TOF profiles in order to determine the mass of measured ions if a number of species exist simultaneously (Yokota et al., 2005). Tables 3 and 4 summarize the specifications of IMA and IEA, respectively.

\section{Instrument Calibration}

We have calibrated the PACE sensors by putting the sensors in a vacuum chamber one by one and injecting nitrogen ions. In order to calibrate IMA, we have also used hydrogen, helium, carbon, oxygen, and heavier ions such as sodium and chlorine to obtain the time profile of the TOF analyzed ions. Figure 4 shows the schematic configuration of the calibration experiment. The sensor under calibration is installed on a rotation table that has a rotation axis parallel to the sensor's axis of rotational symmetry. The rotation ta-

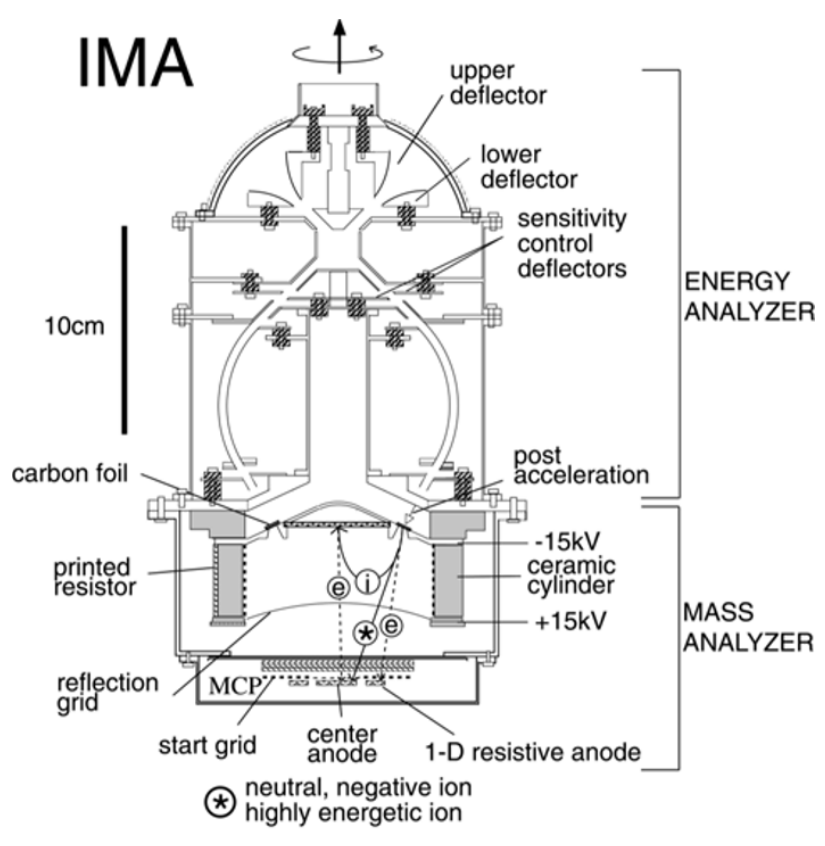

Fig. 2. Cross section of IMA.

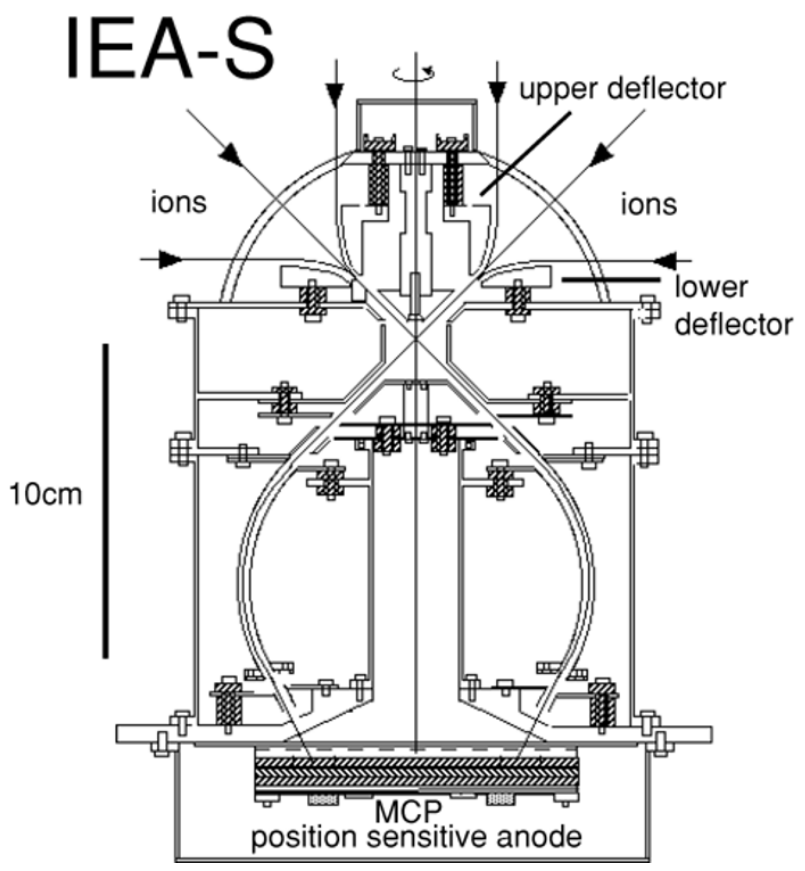

Fig. 3. Cross section of IEA.

ble with the sensor under calibration is installed on another rotation table that has a rotation axis perpendicular to the other rotation axis. The ion beam profile is intermittently monitored by an MCP with a position-sensitive anode that is installed on a movable arm. Most of the data are obtained using 6-keV ion beams since the beam profile is uniform and stable. Lower energy ions $(2 \mathrm{keV})$ are used to measure the performance of the geometrical factor controlling electrode. An analysis of the obtained data confirmed that the PACE sensors are assembled within the mechanical tolerance $(<0.1 \mathrm{~mm})$ and that their characteristics are quite similar to the numerically calculated results. The homogene- 
Table 3. Specifications of IMA

\begin{tabular}{lc}
\hline Energy range & $5 \mathrm{eV} / \mathrm{q}-28 \mathrm{keV} / \mathrm{q}$ \\
\hline Energy resolution & $5 \%(\mathrm{FWHM})$ \\
\hline Energy sweep step & 32 \\
\hline Mass range & $1-60$ \\
\hline Mass resolution & $\mathrm{m} / \Delta \mathrm{m} \sim 15$ \\
\hline Field of view & $2 \pi$ str. \\
\hline FOV sweep range & $45^{\circ} \pm 45^{\circ}(\mathrm{Pol})$ \\
\hline Angular resolution & $5^{\circ}(\mathrm{Pol}) \times 10^{\circ}(\mathrm{Az})(\mathrm{FWHM})$ \\
\hline g-factor $\left(5^{\circ} \times 225^{\circ}\right)$ & $10^{-6} \sim 10^{-4} \mathrm{~cm}^{2} \mathrm{str} \mathrm{keV} / \mathrm{keV}(\mathrm{variable})$ \\
$($ efficiency is not included $)$ & \\
\hline Time resolution & $1 \mathrm{~s}$ \\
\hline Analyzer type & Spherical analyzer \\
Average radius & $55 \mathrm{~mm}$ \\
Gap & $4 \mathrm{~mm}$ \\
\hline
\end{tabular}

Table 4. Specifications of IEA.

\begin{tabular}{lc}
\hline Energy range & $5 \mathrm{eV} / \mathrm{q}-28 \mathrm{keV} / \mathrm{q}$ \\
\hline Energy resolution & $5 \%(\mathrm{FWHM})$ \\
\hline Energy sweep step & 32 \\
\hline Field of view & $2 \pi \mathrm{str}$. \\
\hline FOV sweep range & $45^{\circ} \pm 45^{\circ}(\mathrm{Pol})$ \\
\hline Angular resolution & $5^{\circ}(\mathrm{Pol}) \times 5^{\circ}(\mathrm{Az})(\mathrm{FWHM})$ \\
\hline g-factor $\left(5^{\circ} \times 22.5^{\circ}\right)$ & $10^{-6} \sim 10^{-4} \mathrm{~cm}^{2}$ str keV/keV (variable $)$ \\
$($ efficiency is not included $)$ & $1 \mathrm{~s}$ \\
\hline Time resolution & $\mathrm{Spherical} \mathrm{analyzer}$ \\
\hline Analyzer type & $55 \mathrm{~mm}$ \\
Average radius & $4 \mathrm{~mm}$ \\
Gap &
\end{tabular}

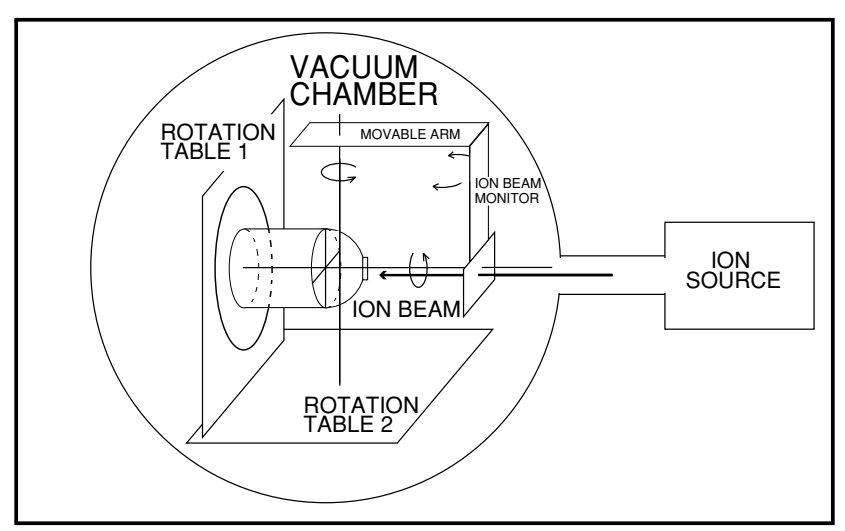

Fig. 4. A schematic diagram that shows the configuration of the calibration experiment.

ity of the analyzer response is also confirmed to be within the acceptable range $(<5 \%)$. Since the absolute on-ground calibration is quite difficult, we are planning to deduce the absolute efficiency of the PACE sensors by the in-flight calibration. We will calculate velocity moments, including density from the data obtained by the PACE sensors. Since it is expected that the cut-off frequency of the continuum radiation corresponds with the surrounding plasma density, we will compare the density calculated from the PACE sensors with the density measured by the plasma wave instrument (LRS) onboard SELENE.

\section{Observation Mode of PACE}

Charged particle signals detected by the PACE sensors are counted and processed by a common electronics box called "MAP-E". An onboard computer in MAP-E processes the obtained count data, and the processed data are stored in an onboard data recorder until they are downloaded to the ground station. Telemetry rate of $42 \mathrm{kbps} / 24 \mathrm{~h}$ are allocated to the PACE sensors. Since the maximum data rate of the PACE sensors are much more than the allocated telemetry rate, the onboard computer will have to process the obtained data. The onboard data processing includes (1) pitch angle sorting of the electron data, (2) sampling the solar wind data by detecting the solar wind direction, (3) data sorting according to the observation mode, and (4) data compression using arithmetic compression code.

In order to realize the optimized observations of the lunar alkali ions, lunar magnetic anomalies, solar wind, and Earth's magnetosphere, PACE has several observation modes. Table 5 summarizes the observation modes with their corresponding data quantity and time resolution.

Since the solar wind ion flux is much higher than that of lunar ions, PACE has a solar wind mode and wake mode (see observation modes No. 6, 8, 9, 10, and 11). In the solar wind mode, IEA selects 128 angular directions centered by the flow direction of ions. The geometrical factor of IEA is electrically reduced to $1 / 50$ in the solar wind mode. The onboard computer can automatically switch the solar wind mode and wake mode according to the intensity of the onboard-calculated ion flux. Two thresholds are used to determine which mode is selected. One of the two thresholds is used to switch from solar wind mode to wake mode, and the other threshold is used to switch from wake mode to solar wind mode. By controlling the levels of these two thresholds, the solar wind mode and wake mode can be switched smoothly. Though both IEA and IMA have a function to reduce the geometrical factor electrically, we are planning to reduce the geometrical factor of IEA only. Since IMA will always look down on the lunar surface, the duration that IMA observes the solar wind is limited. In addition, the alkali ions originating from the lunar surface will always come from the Moon side, so the ion sensor that observes these alkali ions is IMA. Therefore, we expect that in most of the cases, we can observe lunar alkali ions simultaneously with solar wind ions by reducing the geometrical factor of IEA only. The reduced geometrical factor is applied to the entire angular-sweep cycle with the ions below $3 \mathrm{keV} / \mathrm{q}$, including the main component of the solar wind. In order to realize the high spatial resolution of the Electron Reflectometer, PACE has several ER modes (see observation modes No. 7, 8, 9, 10, and 11). In the ER modes, four selected energies are used to measure pitch angle distribution of electrons. Onboard pitch angle sorting is used in the solar wind where the highest time resolution is required due to the existence of rapid variations of the magnetic field direction. When ER modes are used, a three-dimensional (3D) distribution function of electrons with 32 energy steps is also obtained every $60 \mathrm{~s}$. In most of the operation modes, IMA selects eight ion species (see operation modes No. 5, $6,7,8,9)$. In order to measure sputtered ions, PACE has an ion sputtering mode (see observation modes No. 10 and 11). 
Table 5. Observation modes of PACE.

\begin{tabular}{|c|c|c|c|c|c|c|c|}
\hline No. & Mode name & $\begin{array}{l}\text { Solar wind } \\
\text { /Wake }\end{array}$ & $\begin{array}{l}\text { ER } \\
\text { /3D }\end{array}$ & $\begin{array}{l}\text { Electron data } \\
(\mathrm{P} \times \mathrm{Az} \times \mathrm{E})\end{array}$ & $\begin{array}{l}\mathrm{Te} \\
(\mathrm{s})\end{array}$ & $\begin{array}{c}\text { Ion data } \\
(\mathrm{P} \times \mathrm{Az} \times \mathrm{E} \times \mathrm{M})\end{array}$ & $\begin{array}{l}\mathrm{Ti} \\
\text { (s) }\end{array}$ \\
\hline \multirow[t]{2}{*}{1} & TOF test & & $3 \mathrm{D}$ & $32 \times 64 \times 32$ & 16 & IEA: $4 \times 16 \times 32 \times 1$ & 32 \\
\hline & & & & & & IMA: $4 \times 1 \times 32 \times 1024$ & 32 \\
\hline 2 & Position test & & $3 \mathrm{D}$ & $32 \times 64 \times 32$ & 16 & $32 \times 64 \times 32 \times 1$ & 16 \\
\hline 3 & $\begin{array}{c}\text { Magnetosphere } \\
\text { ion } 1 \mathrm{~s}\end{array}$ & & $3 \mathrm{D}$ & $8 \times 16 \times 32$ & 2 & $8 \times 16 \times 32 \times 1$ & 1 \\
\hline 4 & $\begin{array}{c}\text { Magnetosphere } \\
\text { electron } 1 \mathrm{~s}\end{array}$ & & $3 \mathrm{D}$ & $8 \times 16 \times 32$ & 1 & $8 \times 16 \times 32 \times 1$ & 2 \\
\hline \multirow[t]{2}{*}{5} & Magnetosphere & & $3 \mathrm{D}$ & $8 \times 16 \times 32$ & 4 & IEA: $4 \times 16 \times 32 \times 1$ & 16 \\
\hline & backup & & & & & IMA: $4 \times 16 \times 32 \times 8$ & 16 \\
\hline \multirow[t]{4}{*}{6} & Magnetosphere & S & $3 \mathrm{D}$ & $8 \times 16 \times 32$ & 2 & IEA: $128(16 \times 64) \times 32 \times 1$ & 2 \\
\hline & composition & & & & & IMA: $4 \times 16 \times 32 \times 8$ & 8 \\
\hline & & $\mathrm{W}$ & $3 \mathrm{D}$ & $16 \times 64 \times 32$ & 2 & IEA: $16 \times 64 \times 32 \times 1$ & 8 \\
\hline & & & & & & IMA: $4 \times 16 \times 32 \times 8$ & 8 \\
\hline \multirow[t]{2}{*}{7} & ER & & ER & $32(p) \times 4$ & 1 & IEA: $4 \times 16 \times 32 \times 1$ & 16 \\
\hline & backup & & $3 \mathrm{D}$ & $8 \times 16 \times 32$ & 4 & IMA: $4 \times 16 \times 32 \times 8$ & 16 \\
\hline \multirow[t]{4}{*}{8} & ER & $\mathrm{S}$ & ER & $32(\mathrm{p}) \times 4$ & 1 & IEA: $128(16 \times 64) \times 32 \times 1$ & 2 \\
\hline & ION 3D & & $3 \mathrm{D}$ & $8 \times 16 \times 32$ & 2 & IMA: $4 \times 16 \times 32 \times 8$ & 8 \\
\hline & & $\mathrm{W}$ & ER & $16 \times 64 \times 4$ & 1 & IEA: $16 \times 64 \times 32 \times 1$ & 8 \\
\hline & & & $3 \mathrm{D}$ & $8 \times 16 \times 32$ & 4 & IMA: $4 \times 16 \times 32 \times 8$ & 8 \\
\hline \multirow[t]{4}{*}{9} & ER & $\mathrm{S}$ & ER & $32(\mathrm{p}) \times 4$ & 1 & IEA: $128(16 \times 64) \times 32 \times 1$ & 2 \\
\hline & ION 3D & & $3 \mathrm{D}$ & $8 \times 16 \times 32$ & 2 & IMA: $4 \times 16 \times 32 \times 8$ & 8 \\
\hline & 2 & $\mathrm{~W}$ & ER & $32 \times 64 \times 4$ & 2 & IEA: $16 \times 64 \times 32 \times 1$ & 8 \\
\hline & & & $3 \mathrm{D}$ & $8 \times 16 \times 32$ & 4 & IMA: $4 \times 16 \times 32 \times 8$ & 8 \\
\hline \multirow[t]{4}{*}{10} & ER & S & ER & $32(p) \times 4$ & 1 & IEA: $128(16 \times 64) \times 32 \times 1$ & 16 \\
\hline & ION SPUTTER & & $3 \mathrm{D}$ & $8 \times 16 \times 32$ & 2 & IMA: $64(16 \times 64) \times 8 \times 16$ & 16 \\
\hline & & $\mathrm{W}$ & ER & $16 \times 64 \times 4$ & 1 & IEA: $16 \times 64 \times 32 \times 1$ & 16 \\
\hline & & & $3 \mathrm{D}$ & $8 \times 16 \times 32$ & 4 & IMA: $64(16 \times 64) \times 8 \times 16$ & 16 \\
\hline \multirow[t]{4}{*}{11} & ER & $S$ & ER & $32(\mathrm{p}) \times 4$ & 1 & IEA: $128(16 \times 64) \times 32 \times 1$ & 16 \\
\hline & ION SPUTTER & & $3 \mathrm{D}$ & $8 \times 16 \times 32$ & 2 & IMA: $64(16 \times 64) \times 8 \times 16$ & 16 \\
\hline & 2 & W & ER & $32 \times 64 \times 4$ & 2 & IEA: $16 \times 64 \times 32 \times 1$ & 16 \\
\hline & & & $3 \mathrm{D}$ & $8 \times 16 \times 32$ & 4 & IMA: $64(16 \times 64) \times 8 \times 16$ & 16 \\
\hline
\end{tabular}

"S" and "W" in the "Solar wind/Wake" column stand for solar wind mode and wake mode. "P", "Az", "E" and "M" in the "Electron data" and "Ion data" columns stand for polar angle, azimuthal angle, energy and mass. "Te" and "Ti" stand for time resolution of electron measurements and ion measurements, respectively. "(p)" shown in the "Electron data" column stands for pitch angle distribution.

Ions from 64 selected angular directions centered by the solar wind electric field direction with eight selected energies and 16 selected ion species are measured. The solar wind electric field direction that is perpendicular to both the magnetic field direction and solar wind direction is determined by the onboard computer. In order to confirm the validity of time-mass mapping of the TOF measurements, PACE has TOF test mode (see observation mode No. 1). Time profiles obtained by IMA are directly sent without being mapped to selected species in this mode. Highest time resolution in measuring 3-D ion and electron distribution function is achieved by using modes No. 3 and No. 4. Highest angular resolution in measuring 3-D ion and electron distribution function is achieved by using mode No. 2 . In order to realize high time resolution or high angular resolution, no ion mass information is obtained in these modes.

The measured energy of the electrons and ions is determined by the voltage applied to the spherical (IMA and IEA) or toroidal (ESA-S1 and S2) analyzers. The voltage level is stored in a memory, and sequentially D/A converted to apply pre-defined voltage. PACE has multiple sets of sweep energy tables that can be switched by the onboard computer or by commands from the ground. The contents of the sweep energy tables can also be modified by commands from the ground. ESA has eight different sweep energy tables, while IMA and IEA have four different sweep energy tables. Tables 6 and 7 show the energy sweep tables of ESA and IMA/IEA at launch.

\section{Ground Science Data Processing}

The raw telemetry data received on the ground stations are de-packetized and stored in the SELENE database (SELENE Level-1 data). We will process the raw telemetry data of the PACE sensors and produce science data with multiple format (SELENE Level-2 data). This processing includes (1) decompression of the data, (2) correction of the time, (3) subtracting background noise data (if any), and (4) formatting data according to the PACE Level-2 data format. Table 8 shows the PACE Level- 2 science data products that will be stored in the SELENE database. The SELENE database will be open to the public 1 year after the end of the nominal mission operation. 
Table 6. Sweep energy table of ESA.

\begin{tabular}{|c|c|c|c|}
\hline Table no. & Energy range & Sweep wave form & Observation modes \\
\hline 0 & $100 \mathrm{eV}, 200 \mathrm{eV}, 300 \mathrm{eV}, 400 \mathrm{eV}$ & 4 selected energies & $\begin{array}{c}\text { 7-ER, 8W-ER, 8S-ER, 9W-ER, 9S-ER } \\
\text { 10W-ER, 10S-ER 11W-ER, 11S-ER }\end{array}$ \\
\hline 1 & $100 \mathrm{eV}, 300 \mathrm{eV}, 500 \mathrm{eV}, 700 \mathrm{eV}$ & 4 selected energies & $\begin{array}{c}\text { 7-ER, 8W-ER, 8S-ER, 9W-ER, 9S-ER } \\
\text { 10W-ER, 10S-ER 11W-ER, 11S-ER }\end{array}$ \\
\hline 2 & $200 \mathrm{eV}, 400 \mathrm{eV}, 600 \mathrm{eV}, 800 \mathrm{eV}$ & 4 selected energies & $\begin{array}{c}\text { 7-ER, 8W-ER, 8S-ER, 9W-ER, 9S-ER } \\
\text { 10W-ER, 10S-ER 11W-ER, 11S-ER }\end{array}$ \\
\hline 3 & $200 \mathrm{eV}, 500 \mathrm{eV}, 800 \mathrm{eV}, 1 \mathrm{keV}$ & 4 selected energies & $\begin{array}{c}\text { 7-ER, 8W-ER, 8S-ER, 9W-ER, 9S-ER } \\
\text { 10W-ER, 10S-ER 11W-ER, 11S-ER }\end{array}$ \\
\hline 4 & $\begin{array}{l}100 \mathrm{eV}, 200 \mathrm{eV}, 300 \mathrm{eV}, 400 \mathrm{eV} \\
500 \mathrm{eV}, 600 \mathrm{eV}, 700 \mathrm{eV}, 800 \mathrm{eV}\end{array}$ & 8 selected energies & \\
\hline 5 & $\begin{array}{c}100 \mathrm{eV}, 300 \mathrm{eV}, 500 \mathrm{eV}, 700 \mathrm{eV} \\
900 \mathrm{eV}, 1.1 \mathrm{keV}, 1.3 \mathrm{keV}, 1.5 \mathrm{keV}\end{array}$ & 8 selected energies & \\
\hline 6 & $\begin{array}{l}\text { ESA-S1: } 5 \mathrm{eV}-8.676 \mathrm{keV} \\
\text { ESA-S2: } 9 \mathrm{eV}-15.436 \mathrm{keV}\end{array}$ & $\begin{array}{l}32 \text { exponential steps } \\
32 \text { exponential steps }\end{array}$ & $\begin{array}{c}1,2,3,4,5,6 \mathrm{~W}, 6 \mathrm{~S} \\
\text { 7-3D, 8W-3D, 8S-3D, 9W-3D, 9S-3D } \\
\text { 10W-3D, 10S-3D, 11W-3D, 11S-3D }\end{array}$ \\
\hline 7 & $3 \mathrm{eV}-868 \mathrm{eV}$ & 32 exponential steps & $\begin{array}{c}1,2,3,4,5,6 \mathrm{~W}, 6 \mathrm{~S} \\
\text { 7-3D, 8W-3D, 8S-3D, 9W-3D, 9S-3D } \\
\text { 10W-3D, 10S-3D, 11W-3D, 11S-3D }\end{array}$ \\
\hline
\end{tabular}

The column "Observation modes" indicates the observation modes that are permitted to use each table. "W", "S", "ER", and "3D" stand for wake mode, solar wind mode, ER mode and 3D mode, respectively.

Table 7. Sweep energy table of IMA/IEA.

\begin{tabular}{cccc}
\hline Table no. & Energy range & Sweep wave form & Observation modes \\
\hline \hline 0 & IMA: 7 eV-29.154 keV & 32 exponential steps & $1,2,3,4,5,6 \mathrm{~S}, 7,8 \mathrm{~S}, 9 \mathrm{~S}$ \\
& IEA: $300 \mathrm{eV}-8.029 \mathrm{keV}(1 / 50)$ & 32 exponential steps & $1,2,3,4,6 \mathrm{~W}, 8 \mathrm{~W}, 9 \mathrm{~W}$ \\
\hline 1 & IMA: $7 \mathrm{eV}-29.154 \mathrm{keV}$ & 32 exponential steps & $1,2,3,4,10 \mathrm{~W}, 10 \mathrm{~S}, 11 \mathrm{~W}, 11 \mathrm{~S}$ \\
& IEA: $7 \mathrm{eV}-28.680 \mathrm{keV}$ & 32 exponential steps & \\
\hline 2 & IMA: $9 \mathrm{eV}-2 \mathrm{keV}$ & 32 exponential steps & $1,2,3,4$ \\
& IEA: $300 \mathrm{eV}-8.029 \mathrm{keV}(1 / 50)$ & 32 exponential steps & 32 exponential steps \\
\hline
\end{tabular}

The column "Observation modes" indicates the observation modes that are permitted to use each table. "W" and "S" stand for wake mode and solar wind mode, respectively. "(1/50)" shown in the "Energy range" column indicates that the sensitivity is electrically reduced to $1 / 50$.

Table 8. PACE Level-2 science data products.

\begin{tabular}{cccc}
\hline Product name & Description & Format & Time resolution \\
\hline PACE_PBF1 & High resoution count data of electron/ion energy spectrum & BINARY & $1 \mathrm{~s}$ \\
PACE_CDF & High resoution count data of electron/ion energy spectrum & CDF & $1 \mathrm{~s}$ \\
PACE_KP & Key parameter of electron/ion energy spectrum & ASCII & $1 \mathrm{~min}$ \\
PACE_ET_summary & Summary plot of electron/ion E-T diagram & IMAGE & 1 plot/1 day \\
PACE_ERMA_MAP & Magnetic anomaly map (electron reflectometer) & PDS & N.A. \\
PACE_SI_MAP & Secondary ion map & PDS & N.A. \\
\hline
\end{tabular}

\section{Expected PACE Observations \\ 7.1 Observation of alkali ions}

The fact that the Moon, Mercury, some of the planetary satellites and asteroids maintain alkali atmospheres that are mostly thin and sometimes transient has recently received much interest. The Moon and its atmosphere have been the most extensively investigated, and the findings are thought to be the cornerstone on which to build studies of the other celestial bodies. The lunar alkali atmosphere is called a "surface-bounded exosphere" because it is thin enough to be regarded as an exosphere and it bounds on the solid surface differently from the Earth's atmosphere-bounded exosphere. A number of instruments onboard spacecraft which measured electrons, magnetic fields, among other parameters, brought about significant advances in our knowledge of the plasma environment around the Moon. There have been, however, only a few detailed ion measurements around the Moon.

Towards the end of $1980 \mathrm{~s}, \mathrm{D}_{2}$-line emissions from atomic $\mathrm{Na}$ and $\mathrm{K}$, indicating the existence of $\mathrm{Na}$ and $\mathrm{K}$ atmospheres, were discovered above the sunlit limb of the Moon by a ground-based optical observation with long-slit high resolution spectroscopy (Potter and Morgan, 1988) simultaneously with the other discovery of lunar $\mathrm{Na}$ achieved by Tyler et al. (1988). The alkali atoms were the only detectable species, while the estimated surface number densi- 
ties of a few $10 \mathrm{~s} / \mathrm{cm}^{3}$ were very small. Thus, the remote study of the ground-based observations has been carried out actively since then in order to study the dynamics and source processes. We can now expect the existence of a substantial amount of alkali ions originating from the lunar atmosphere and surface. The ions are produced mainly by the desorption of the lunar soil constituents as ions, and photoionization of the atmospheric particles. These are pickedup and lost by the solar wind immediately after they are generated. The measurement of such ions in orbit offers key information on the lunar ionized atmosphere, production and loss mechanisms, distribution, and composition.

We know that the electric and magnetic fields of the solar wind are dominant around the Moon, especially in the upstream of the Moon when the Moon is out of the Earth's magnetosphere, because the Moon has neither a global dipole magnetic field, nor a sufficiently large conductive core to affect the surrounding electric and magnetic fields. Note that we will consider the electric potential and the magnetic anomaly on the lunar surface in future detailed analysis of picked-up lunar ions. Global mapping of the crustal magnetic anomaly on the lunar surface were presented by Hood et al. (2001) and Halekas et al. (2001). The extremely thin lunar atmosphere allows the solar wind to directly reach the lunar surface and to sputter ions reflecting the stoichiometry of the surface. Ions are also generated from the lunar atmospheres by the solar UV. These ions are transported by the solar wind in the cycloidal motion without any collisions with atmospheric particles if the electric and magnetic fields are steady and their initial energies are negligible. Some of the ions picked-up by the solar wind hit the lunar surface and are reabsorbed, and the others are driven to the exterior and reach the lunar orbit. Because the lunar radius and lunar orbital altitude are much shorter than the Larmor radius of picked-up lunar ions, motions of picked-up ions from the production points to the orbital altitude are nearly in the direction of the electric field. Ion kinetic energy that is supplied mainly by the solar wind electric field till orbital altitude is approximately in proportion to the ion flight length provided that the surrounding electric field is nearly constant. Energy spectra of pickedup lunar ions correspond to the spatial distribution of their production. Thus, energy analysis of picked-up lunar ions with simultaneous monitoring of the solar wind electric and magnetic fields lead to the ion production distribution and then to discrimination of the surface origin from the atmosphere origin (see Fig. 5). In addition, mass analysis can distinguish ion species, including $\mathrm{H}^{+}$and $\mathrm{He}^{++}$of the solar wind origin, and $\mathrm{Na}^{+}$and $\mathrm{K}^{+}$of the lunar origin.

Measurement of lunar ionized atmospheric particles enables quasi-continuous monitoring of the thin lunar atmosphere. Ground-based observations, which have advanced the studies of the lunar alkali atmosphere, have a limited window, demanding strict observation conditions. Although the typical orbiter altitude of $100 \mathrm{~km}$ is less than the scale heights (several hundreds of kilometers) of the observed alkali atmospheres, most of these atmospheric particles $(\geq 80 \%)$ exist below $100 \mathrm{~km}$. We note that in-situ ion measurement of picked-up lunar ions on lunar orbiters provides altitudinal distribution above some local area, not

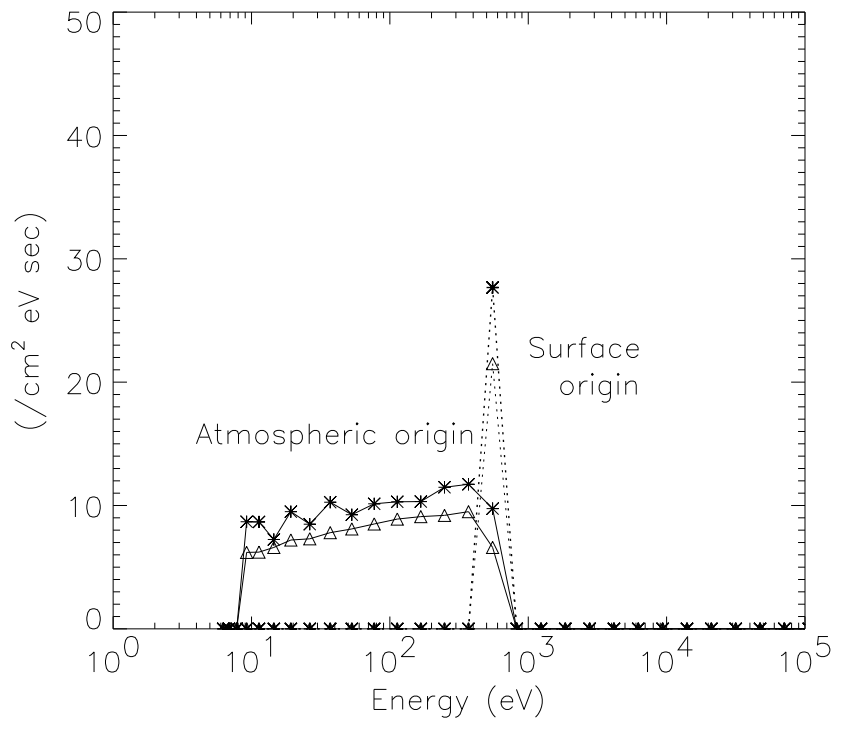

Fig. 5. Energy spectra of picked-up lunar $\mathrm{Na}^{+}$and $\mathrm{K}^{+}$ions at an altitude of $100 \mathrm{~km}$. The asterisk and triangle denote $\mathrm{Na}^{+}$and $\mathrm{K}^{+}$, respectively. Solid and dashed lines denote ions of atmospheric origin and surface origin, respectively.

global distribution. Long-term and continuous monitoring of the lunar atmosphere by using ionized atmospheric particle distribution presumably provides us with a new set of lenses through which to view the production, transport, and loss mechanism of the lunar atmosphere in detail.

In addition, we can distinguish secondary ions sputtered by the solar wind by using the energy spectra of picked-up lunar ions (Yokota et al., 2005). This helps global compositional mapping of the lunar surface by measuring sputtered ions, which is called Remote SIMS. The Remote SIMS measurement by a lunar orbiter certainly complements Xray fluorescence and gamma-ray spectroscopy for elemental abundance and multi-band spectral imaging for mineralogy (Elphic et al., 1991).

The plasma environment around the Moon is similar to those around the other planets, satellites, and asteroids which have a weak magnetic field and thin atmosphere-for example, Io, and Europa. The first trial to observe lunar alkali ions in the lunar orbit will certainly advance the whole planetary atmospheric science.

\subsection{Observation of magnetic anomalies}

In order to verify the optimality of the PACE observation modes for detecting magnetic anomalies on the lunar surface, we estimated the error of detecting magnetic field intensity using a model dipole magnetic anomaly on the lunar surface. PACE-ESA sensors observe a 3-D distribution function of electrons by dividing the spherical field of view into 32 polar angles $\times 64$ azimuthal angles (in the highest angular resolution mode) in the polar coordinate system whose poles are pointed to/from the lunar surface. The observed energy range is divided into 32 steps in the maximum energy steps mode. Figure 6 shows the model dipole magnetic anomaly and the magnetic line of force when both the external magnetic filed and the dipole magnetic moment is perpendicular to the lunar surface. In calculating the pitch angle distribution of the incident electrons, we made the 


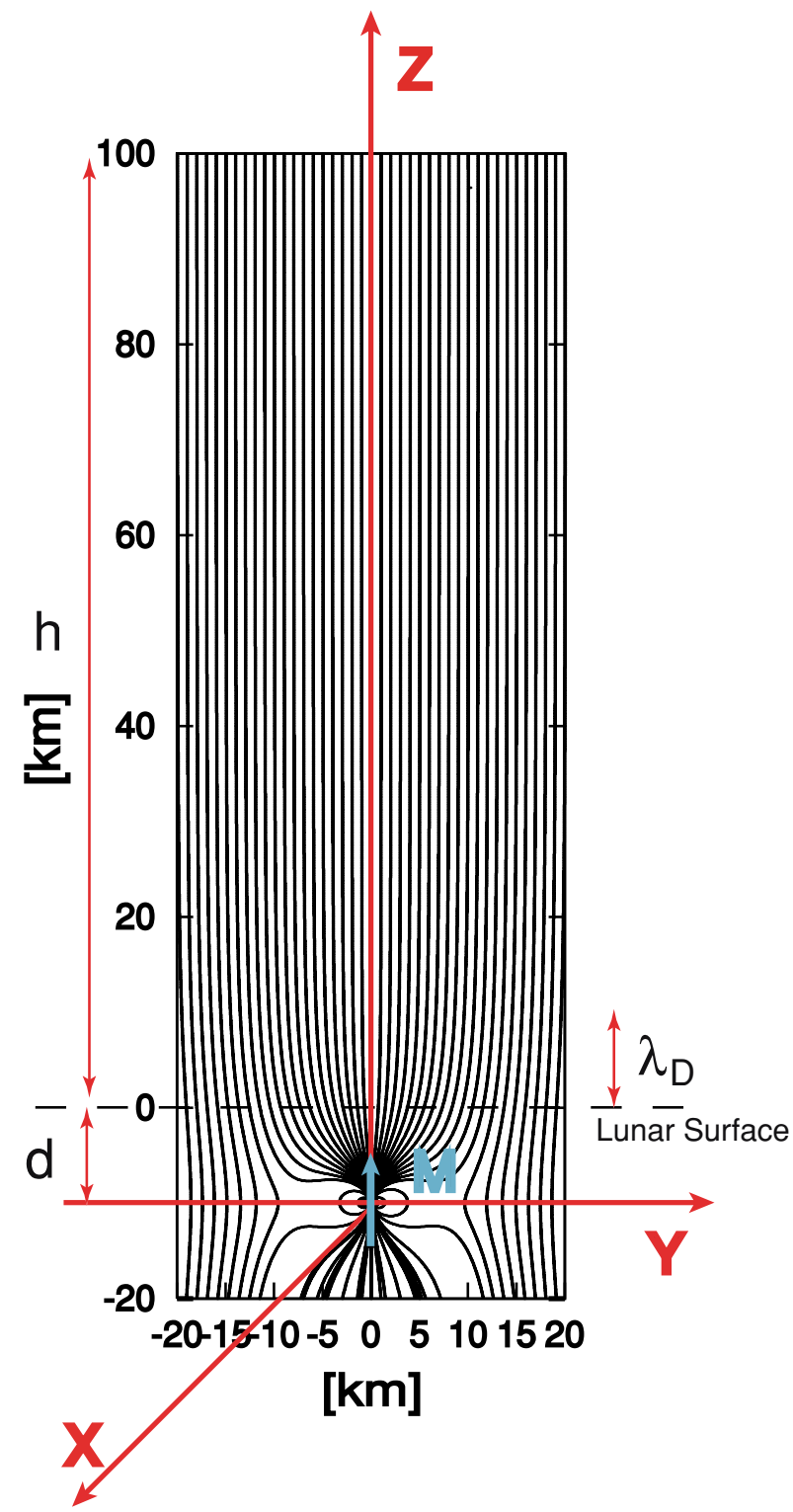

Fig. 6. Model magnetic anomaly on the lunar surface.

following assumptions: (1) the altitude of SELENE was $100 \mathrm{~km}$; (2) the depth of the dipole magnetic moment $d$ was $10 \mathrm{~km}$; (3) the intensity of the dipole magnetic moment was $5.0 \times 10^{10} \mathrm{~A} \mathrm{~m}^{2}$ where the magnetic anomaly on the lunar surface was $10 \mathrm{nT}$ when the direction of the dipole moment was shown in Fig. 6; (4) the potential difference between lunar surface and SELENE was assumed to be the typical value of $-50 \mathrm{~V}$ in the night side (Halekas et al., 2001); (5) the external magnetic field of $10 \mathrm{nT}$ directed to $-\mathrm{Z}$ direction (typical magnetic field intensity in the lobe region); (6) Debye length was $525 \mathrm{~m}$ where electron density was $0.01 \mathrm{~cm}^{-3}$, electron temperature was $50 \mathrm{eV}$ (typical value in the lobe region). Figure 7 shows the calculated pitch angle distribution of electrons detected by a model electron sensor that simulates the characteristics of ESA-S1. We obtained the pitch angle distribution by using the averaged electron flux in the night side region observed by Lunar Prospector. The assumed sampling time of electron measurements was $32 \mathrm{~ms}$. We also assumed that the fluctuation of the count
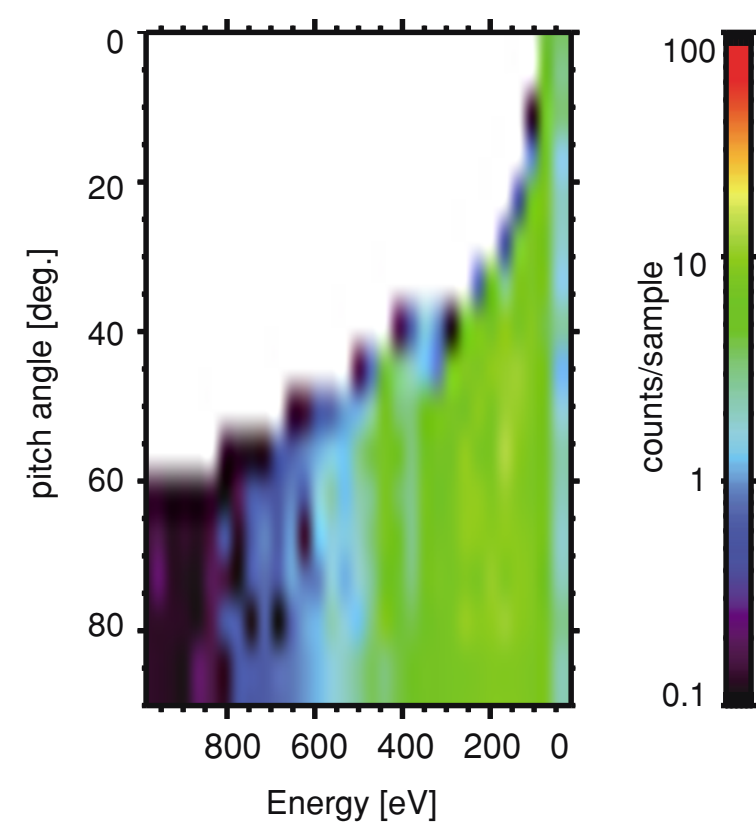

Fig. 7. Calculated pitch angle distribution of electrons.

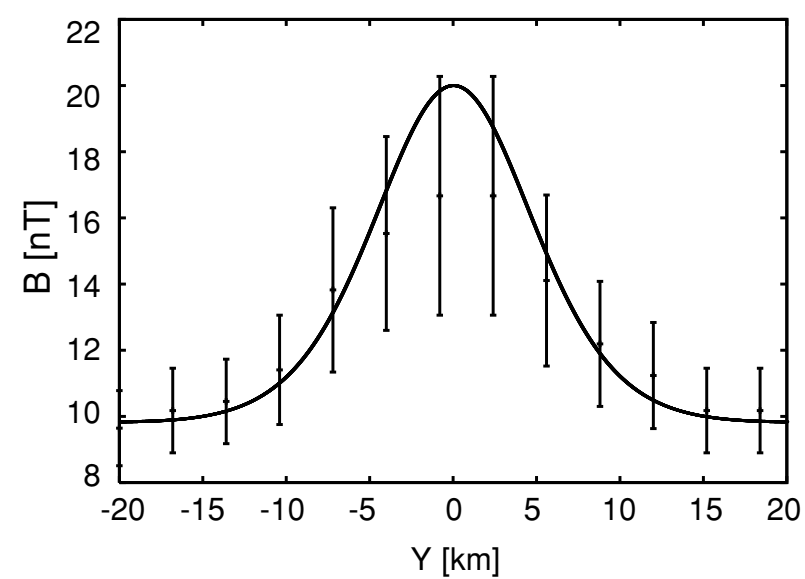

Fig. 8. Reproduced intensity of the magnetic anomaly when the number of measured energy steps was four and the number of polar angular division was 16 .

rate obeyed the Poisson distribution.

We simulated the electron reflectometer measurement by sampling the pitch angle distribution at discrete 4 (78$203 \mathrm{eV}), 8(78-453 \mathrm{eV}), 16(78-703 \mathrm{eV})$, and 32 (16$984 \mathrm{eV}$ ) energy steps. Since the external magnetic field is assumed to be parallel to the axis of symmetry of the ESA sensors, the pitch angle was sampled according as the polar angular division of the ESA sensors. Figure 8 shows the result of the simulated electron reflectometer measurement when the four discrete energy steps were measured and the polar angular division was 16 . The measured magnetic anomaly intensity is indicated by cross marks with error bars. The modeled magnetic anomaly is also shown by a solid line. Since it took $2 \mathrm{~s}$ to obtain four energy steps and 16 pitch angle distributions, the intensity of the magnetic anomaly was obtained every $3.2 \mathrm{~km}$ along the orbit of the SELENE satellite, taking into account the orbital speed of the SELENE satellite of $\sim 1.6 \mathrm{~km} / \mathrm{s}$. The peak intensity 


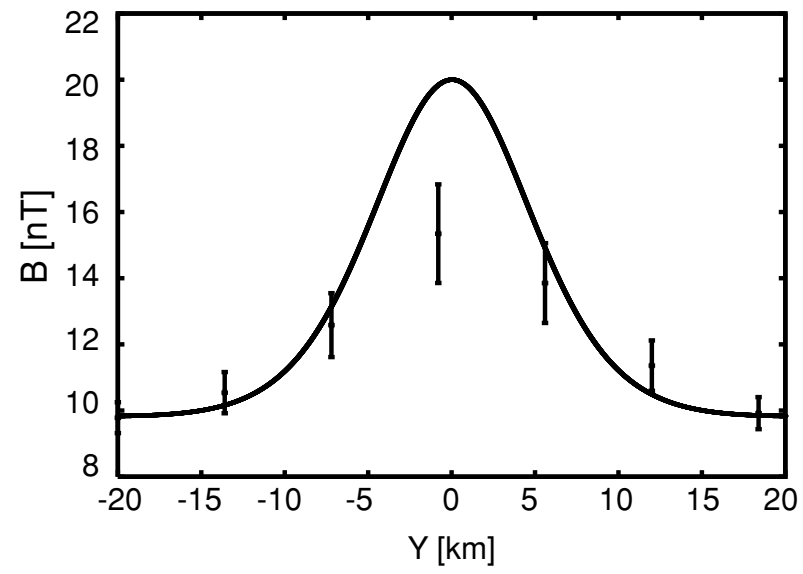

Fig. 9. Reproduced intensity of the magnetic anomaly when the number of measured energy steps was eight and the number of polar angular division was 16 .

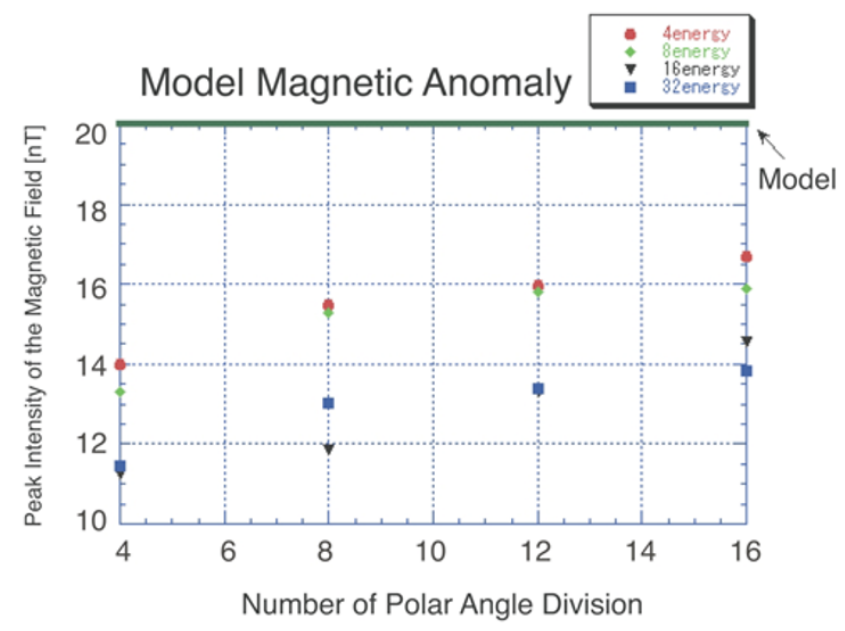

Fig. 10. Peak intensity of the measured magnetic anomaly with respect to the number of the polar angle division of $4,8,12$ and 16 . The estimated magnetic anomaly for the energy steps of $4,8,16$, and 32 are shown.

of the obtained magnetic anomaly was less than the modeled intensity because the measured intensity was averaged within the Larmor motion of the electrons. This effect also resulted in the higher intensity of the measured magnetic anomaly than the modeled anomaly in the surrounding region. Figure 9 shows the result of the simulated electron reflectometer measurement when the eight discrete energy steps were measured and the polar angular division was 16 . Since it took $4 \mathrm{~s}$ to obtain eight energy steps and 16 pitch angle distributions, the spatial resolution of the magnetic anomaly measurement was lower by a factor of two than the previous case. The error bar was smaller than the previous case because the number of the data used for determining the intensity was twice larger. Figure 10 shows the peak intensity of the measured magnetic anomaly with respect to the number of the polar angle division of 4, 8, 12, and 16 . The estimated magnetic anomaly for the energy steps of 4 , 8,16 , and 32 are shown. The simulated results indicate that it is quite important to select the energy as low as possible to reproduce the intensity of the magnetic anomaly. The result also indicated that as long as the appropriate energy was se-
Table 9. Spatial resolution of the lunar magnetic anomaly detection.

\begin{tabular}{c|rrrc}
\hline & \multicolumn{1}{|c}{$80[\mathrm{eV}]$} & $100[\mathrm{eV}]$ & $150[\mathrm{eV}]$ & $300[\mathrm{eV}]$ \\
\hline $0.0625[\mathrm{~s}]$ & $6.55[\mathrm{~km}]$ & $7.21[\mathrm{~km}]$ & $8.66[\mathrm{~km}]$ & $11.99[\mathrm{~km}]$ \\
$1.0[\mathrm{~s}]$ & $6.74[\mathrm{~km}]$ & $7.39[\mathrm{~km}]$ & $8.80[\mathrm{~km}]$ & $12.09[\mathrm{~km}]$ \\
$4.0[\mathrm{~s}]$ & $9.16[\mathrm{~km}]$ & $9.64[\mathrm{~km}]$ & $10.77[\mathrm{~km}]$ & $13.59[\mathrm{~km}]$ \\
$16.0[\mathrm{~s}]$ & $26.42[\mathrm{~km}]$ & $26.60[\mathrm{~km}]$ & $27.02[\mathrm{~km}]$ & $28.27[\mathrm{~km}]$ \\
\hline
\end{tabular}

lected, the number of the energy steps could be as small as four. Concerning the number of the polar angular division, larger than eight is desirable since the reproduced intensity is quite different between the number of polar angular division of four and eight.

In order to realize the high spatial resolution measurements, the number of the measured energy steps and the number of the polar angular division should be as small as possible. The spatial resolution $\Delta$ of detecting the magnetic field anomalies using Electron Reflectometer is expressed as follows (Lin et al., 1976): $\Delta \sim\left(R_{e}^{2}+\left(2 r_{L}\right)^{2}+l^{2}\right)^{\frac{1}{2}}$ where $l$ is the distance of the satellite orbital motion during the measurement and $r_{L}$ is the Larmor radius of measured electrons. $R_{e}$ is expressed using the altitude of the spacecraft $h$, the angle between the external magnetic field and the direction normal to the lunar surface $\phi$, and the error in determining the external magnetic field measured by a magnetometer on the spacecraft $\delta$ as follows: $R_{e} \simeq \delta h \sec ^{2} \phi$. When the accuracy of the magnetic field measurement is $0.1 \mathrm{nT}$, and the intensity of the external magnetic field is $10 \mathrm{nT}, \delta$ is $0.6^{\circ}$. Assuming that $|\phi| \leq 60^{\circ}$ and $h=100 \mathrm{~km}$, $R_{e}=1 \sim 4 \mathrm{~km}$. The orbital motion of the SELENE satellite is $\sim 1.6 \mathrm{~km} / \mathrm{s}$. Table 9 shows the estimated spatial resolution of detecting the magnetic field anomalies using the Electron Reflectometer with respect to the time resolution of the electron measurement and the measured electron energy.

Based on the results of our reproduction of the modeled magnetic anomalies, we can conclude that the observation mode of ESA sensors optimized for high-spatial resolution electron reflectometer should have four discrete energy steps that are as low as possible and eight polar angular divisions. With this observation mode, we will be able to measure the magnetic anomaly with time resolution of $1 \mathrm{~s}$ that amounts to the spatial resolution of below $10 \mathrm{~km}$.

\section{Conclusion}

One of the scientific instruments on SELENE, PACE, will measure 3-D distribution function of low-energy ions and electrons at an altitude of $100 \mathrm{~km}$ around the Moon. With the minimum time resolution of $1 \mathrm{~s}$, high spatial resolution measurement of magnetic anomalies on the lunar surface will be made. Since no one has as yet measured the 3-D distribution function of low-energy ions at $100 \mathrm{~km}$ altitude around the Moon, out of the equatorial plane, it is expected that many unresolved problems concerning the lunar plasma environment will be elucidated by the PACE observation.

Acknowledgments. The authors wish to express their sincere thanks to all the members of SELENE project team for their supports in developing the PACE sensors. The authors also wish to express their grateful thanks to Dr. Yasuo Arai of High Energy 
Accelerator Research Organization for providing TDC chips that were indispensable for the TOF measurement of IMA. SELENEPACE sensors were manufactured by Mitaka Kohki Co. Ltd., Meisei Elec. Co., Hamamatsu Photonics K.K., and Kyocera Co.

\section{References}

Anderson, K. A. and D. E. Wilhelms, Correlation of Lunar Far-Side Magnetized Regions with Ringed Impact Basins, Earth Planet. Sci. Lett., 46, 107-112, 1979.

Anderson, K. A., L. M. Chase, R. P. Lin, J. E. McCoy, and R. E. McGuire, Solar-wind and interplanetary electron measurements on the Apollo 15 subsatellite, J. Geophys. Res., 77, 4611-4626, 1972.

Anderson, K. A., R. P. Lin, R. E. McGuire, and J. E. McCoy, Measurement of Lunar and Planetary Magnetic Fields by Reflection of Low Energy Electrons, Space Sci. Insr., 1, 439-470, 1975.

Anderson, K. A., R. P. Lin, R. E. McGuire, J. E. McCoy, C. T. Russell, and P. J. Coleman, Linear Magnetization Feature Associated with Rima Sirsalis, Earth Planet. Sci. Lett., 34, 141-151, 1977.

Binder, A. B., Lunar Prospector: Overview, Science, 281, 1475-1476, 1998.

Clay, D. R., B. E. Goldstein, M. Neugebauer, and C. W. Snyder, Solar-wind spectrometer experiment, Apollo 15 Preliminary Science Report, NASA Spec. Publ., 289, 10-1-10-7, 1972.

Colburn, D. S., R. G. Currie, J. D. Mihalov, and C. P. Sonett, Diamagnetic solar-wind cavity discovered behind Moon, Science, 158, 1040-1042, 1967.

Criswell, D. R., Photoelectrons and solar wind/lunar limb interaction, The Moon, 7, 202-238, 1973.

Elphic, R. C., H. O. Funsten, B. L. Barraclough, D. J. McComas, M. T. Paffett, D. T. Vaniman, and G. Heiken, Lunar surface composition and solar wind-induced secondary ion mass spectrometry, Geophys. Res. Lett., 18, 2165-2168, 1991.

Foing, B. H., G. D. Racca, A. Marini, E. Evrard, L. Stagnaro, M. Almeida, D. Koschny, D. Frew, J. Zender, J. Heather, M. Grande, J. Huovelin, H. U. Keller, A. Nathues, J. L. Josset, A. Malkki, W. Schmidt, G. Noci, R. Birkl, L. Iess, Z. Sodnik, and P. McManamon, SMART-1 mission to the Moon: Status, first results and goals, Adv. Space Res, 37, 6-13, 2006.

Futaana, Y., S. Machida, Y. Saito, A. Matsuoka, and H. Hayakawa, Counterstreaming electrons in the near vicinity of the Moon observed by plasma instruments on board NOZOMI, J. Geophys. Res., 106, 1872918740, 2001

Futaana, Y., S. Machida, Y. Saito, A. Matsuoka, and H. Hayakawa, Moonrelated nonthermal ions observed by Nozomi: Species, sources, and generation mechanisms, J. Geophys. Res., 108(A1), 1025, doi:10.1029/ 2002JA009366, 2003.

Halekas, J. S., D. L. Mitchell, R. P. Lin, S. Frey, L. L. Hood, M. H. Acuña, and A. B. Binder, Mapping of crustal magnetic anomalies on the lunar near side by the Lunar Prospector electron reflectometer, J. Geophys. Res., 106(E11), 27,841-27,852, 2001.

Hilchenbach, M., D. Hovstadt, B. Klecker, and E. Möbius, Observation of energetic lunar pick-up ions near earth, Adv. Space Res., 13, 321-324, 1993.

Hills, H. K., J. C. Meister, R. R. Vondrak, and J. W. Freeman, Jr, Suprathermal ion detector experiment, Apollo 15 Preliminary Science Report, NASA Spec. Publ., 289, 12-1-12-10, 1972.

Hood, L. L., A. Zakharian, J. Halekas, D. L. Mitchell, R. P. Lin, M. H. Acuña, and A. B. Binder, Initial mapping and interpretation of lunar crustal magnetic anomalies using Lunar Prospector magnetometer data,
J. Geophys. Res., 106(E11), 27,825-27,840, 2001.

Howe, H. C., R. P. Lin, R. E. McGuire, and K. A. Anderson, Energetic Electron Scattering from the Lunar Remanent Magnetic Field, Geophys. Res. Lett., 1, 101-104, 1974.

Lin, R. P., A. Anderson, R. Bush, and R. E. McGuire, Lunar surface remnant magnetic fields detected by the electron reflection method, Proc. Lunar Sci. Conf. 7th, 2691-2703, 1976.

Lin, R. P., D. L. Mitchell, D. W. Curtis, K. A. Anderson, C. W. Carlson, J. McFadden, M. H. Acuna, L. L. Hood, and A. Binder, Lunar surface magnetic fields and their interaction with the solar wind: results from Lunar Prospector, Science, 281, 1480-1484, 1998.

Lyon, E. F., H. S. Bridge, and J. H. Binsack, Explorer 35 plasma measurements in the vicinity of the Moon, J. Geophys. Res., 72, 6113-6117, 1967.

Mall, U., E. Kirsch, K. Cierpka, B. Wilken, A. Söding, F. Neubauer, G. Gloeckler, and A. Galvin, Direct observation of lunar pick-up ions near the Moon, Geophys. Res. Lett., 25(20), 3799-3802, 1998.

McComas, D. J. and J. E. Nordholt, New approach to 3-D, high sensitivity, high mass resolution space plasma composition measurements, Rev. Sci. Instrum., 61, 3095-3097, 1990.

Neugebauer, M., C. W. Snyder, D. R. Clay, and B. E. Goldstein, Solar wind observations on the lunar surface with the Apollo-12 ALSEP, Planet. Space Sci., 20, 1577-1591, 1972.

Nozette, S., P. Rustan, L. P. Pleasance, J. F. Kordas, I. T. Lewis, H. S. Park, R. E. Priest, D. M. Horan, P. Regeon, C. L. Lichtenberg, E. M. Shoemaker, E. M. Eliason, A. S. McEwen, M. S. Robinson, P. D. Spudis, C. H. Acton, B. J. Buratti, T. C. Duxbury, D. N. Baker, B. M. Jakosky, J. E. Blamont, M. P. Corson, J. H. Resnick, C. J. Rollins, M. E. Davies, P. G. Lucey, E. Malaret, M. A. Massie, C. M. Pieters, R. A. Reisse, R. A. Simpson, D. E. Smith, T. C. Sorenson, R. W. Vorder Breugge, and M. T. Zuber, The Clementine Mission to the Moon: Scientific Overview, Science, 266, 1835-1843, 1994.

Ogilvie, K. W., J. T. Steinberg, R. J. Fitzenreiter, C. J. Owen, A. J. Lazarus, W. J. Farrell, and R. B. Torbert, Observations of the lunar plasma wake from the WIND spacecraft on December 27, 1994, Geophys. Res. Lett., 23, 1255-1258, 1996.

Potter, A. E. and T. H. Morgan, Discovery of the sodium and potassium vapor in the atmosphere of the Moon, Science, 241, 675-679, 1988.

Potter, A. E., R. M. Killen, and T. H. Morgan, Variation of lunar sodium during passage of the Moon through the Earth's magnetotail, J. Geophys. Res., 105, 15073-15084, 2000.

Stern, S. A., The lunar atmosphere: history, status, current problems, and context, Rev. Geophys., 37, 453-491, 1999.

Tyler, A. L., W. H. Kozlowski, and D. M. Hunten, Observations of sodium in the tenuous lunar atmosphere, Geophys. Res. Lett., 15, 1141-1144, 1988.

Yokota, S. and Y. Saito, Estimation of picked-up lunar ions for future compositional remote SIMS analyses of the lunar surface, Earth Planets Space, 57, 281-289, 2005.

Yokota, S., Y. Saito, K. Asamura, and T. Mukai, Development of an ion energy mass spectrometer for application on board three-axis stabilized spacecraft, Rev. Sci. Instrum., 76, 014501-1-014501-8, 2005.

Y. Saito (e-mail: saito@stp.isas.jaxa.jp), S. Yokota, K. Asamura, T. Tanaka, R. Akiba, M. Fujimoto, H. Hasegawa, H. Hayakawa, M. Hirahara, M. Hoshino, S. Machida, T. Mukai, T. Nagai, T. Nagatsuma, M. Nakamura, K.-i. Oyama, E. Sagawa, S. Sasaki, K. Seki, and T. Terasawa 